\title{
Coupling AAV-mediated promoterless gene targeting to SaCas9 nuclease to efficiently correct liver metabolic diseases
}

\author{
Alessia De Caneva, ${ }^{1}$ Fabiola Porro, ${ }^{1}$ Giulia Bortolussi, ${ }^{1}$ Riccardo Sola, ${ }^{1}$ Michela Lisjak, ${ }^{1}$ Adi Barzel, ${ }^{2}$ \\ Mauro Giacca, ${ }^{1}$ Mark A. Kay, ${ }^{3}$ Kristian Vlahoviček, ${ }^{4}$ Lorena Zentilin, ${ }^{1}$ and Andrés F. Muro ${ }^{1}$ \\ ${ }^{1}$ International Centre for Genetic Engineering and Biotechnology (ICGEB), Trieste, Italy. ${ }^{2}$ Cancer Biology Research Center, \\ Tel Aviv University, Tel Aviv, Israel. ${ }^{3}$ Departments of Pediatrics and Genetics, Stanford University, Stanford, California, USA. \\ ${ }^{4}$ Bioinformatics Group, Division of Molecular Biology, Department of Biology, Faculty of Science, University of Zagreb, \\ Zagreb, Croatia.
}

Nonintegrative AAV-mediated gene therapy in the liver is effective in adult patients but faces limitations in pediatric settings because of episomal DNA loss during hepatocyte proliferation. Gene targeting is a promising approach as it results in the permanent modification of the genome. We previously rescued neonatal lethality in Crigler-Najjar mice by inserting a promoterless human uridine glucuronosyl transferase A1 (UCT1A1) cDNA in exon 14 of the albumin gene, without the use of nucleases. To increase the recombination rate and therapeutic efficacy, we used CRISPR/SaCas9. Neonatal mice were transduced with 2 AAVs: one expressing the SaCas9 and sgRNA and one containing a promoterless cDNA flanked by albumin homology regions. Targeting efficiency increased approximately 26 -fold with an EGFP reporter CDNA, reaching up to $24 \%$ of EGFP-positive hepatocytes. Next, we fully corrected the diseased phenotype of Crigler-Najjar mice by targeting the hUGT1A1 cDNA. Treated mice had normal plasma bilirubin up to 10 months after administration, hUGT1A1 protein levels were approximately 6 -fold higher than in WT liver, with a 90 -fold increase in recombination rate. Liver histology, inflammatory markers, and plasma albumin were normal in treated mice, with no off-targets in predicted sites. Thus, the improved efficacy and reassuring safety profile support the potential application of the proposed approach to other liver diseases.

Conflict of interest: $M A K$ and $A B$ are scientific cofounders and are on the board of directors of LogicBio Therapeutics, which has licensed the GeneRide technology from Stanford University. CB and AFM are inventors of a patent describing AAV-mediated liver gene transfer (treatment of hyperbilirubinemia, CA2942451).

Copyright: (c) 2019, American Society for Clinical Investigation.

Submitted: March 18, 2019

Accepted: June 13, 2019

Published: August 8, 2019.

Reference information: JCl Insight. 2019;4(15):e128863. https://doi. org/10.1172/jci.insight.128863.

\section{Introduction}

The liver carries out a wide variety of vital functions, including metabolism of proteins, lipids, and carbohydrates; synthesis of plasma proteins, such as albumin and clotting factors; formation and secretion of bile; and detoxification of body metabolites, such as ammonia and bilirubin. Mutations of genes responsible for these functions cause different disorders, many of which manifest within the first weeks after birth, resulting in the risk of developing permanent damage or death of the patient $(1,2)$. No effective and permanent cure exists for most of these disorders, except for orthotopic liver transplantation (OLT), which is not applicable in all cases because of a shortage of compatible organs. Despite the constant progress in OLT in the last few years, it remains a very invasive procedure with substantial risks and shortcomings (3), such as rejection and life-long immunosuppression, with the threat for the development of de novo malignancies, cardiovascular complications, and infections (4-7). These concerns and limitations emphasize the clinical need to find alternative therapies to cure liver-related genetic disorders.

Gene therapy approaches have the potential to cure monogenic liver diseases, as recently underscored in hemophilia clinical trials in adult patients using AAV-mediated liver gene transfer (8-11). However, a major concern emerges when considering the neonatal/pediatric setting because the progressive loss of episomal AAV viral genomes over time, in concert with hepatocyte proliferation, results in the reduction of both transgene expression and efficacy of the treatment (12-14). Importantly, readministration of the therapeutic vector later in time may not be possible because of the presence of anti-AAV-neutralizing antibodies generated after the first vector administration (15-18). These obstacles highlight the clinical need to develop novel long-lasting therapies for the treatment of neonatal and pediatric liver-related genetic disorders. Therefore, the development of effective approaches permanently modifying the genome of diseased hepatocytes is a highly desirable therapeutic option. 
We recently developed the GeneRide approach, a very promising methodology based on the insertion of a therapeutic cDNA into the albumin locus without the use of nucleases and without disrupting the albumin gene (19). In this approach, a promoterless therapeutic cDNA is spontaneously inserted in-frame just upstream of the albumin stop codon, resulting in a fused mRNA that is translated into 2 separated proteins: albumin and the therapeutic one. The targeted allele continues to actively produce albumin and is stably transmitted to daughter cells without loss of genetic information. We have successfully applied the GeneRide strategy to a lethal mouse model of Crigler-Najjar syndrome type I (CNSI), showing the complete rescue of neonatal lethality and cerebellar and behavioral abnormalities (20). However, plasma bilirubin levels, despite being stable, safe, and life-compatible even 12 months after AAV delivery, were not fully normalized, prompting us to improve the gene-targeting rate and the therapeutic potential of the approach.

The use of engineered endonucleases (21) to increase the homology-directed repair (HDR) rate (22) made site-specific genome modifications at the reach of therapeutic potential. Among the programmable nucleases, the CRISPR/Cas9 platform, based on RNA-guided DNA cleavage, has demonstrated high versatility and efficacy to edit genes both in vitro and in vivo (23-27). We present here what we believe to be a novel, versatile, safe, and powerful therapeutic approach based on the combination of GeneRide (19) with CRISPR/ SaCas9-mediated genome editing (28) in a lethal mouse model of a severe liver disease (29, 30). These data represent the proof of principle showing that the strategy could be used to rescue several genetic metabolic disorders affecting the liver.

\section{Results}

Genome targeting of the albumin locus with RNA-guided SaCas 9 nuclease. To increase the therapeutic efficacy of liver gene-targeting into the albumin locus, we combined the GeneRide methodology $(19,20)$ with the CRISPR/ SaCas9 platform (ref. 28 and Figure 1, A and B). The strategy is based on the simultaneous delivery of 2 rAAV8 vectors: one contains the donor DNA and the promoterless therapeutic CDNA, whereas the other one expresses both the $\mathrm{SaCas} 9$ and the sgRNA under the transcriptional control of liver-specific and U6 promoters, respectively. The marked liver tropism of the AAV serotype 8 (31) guarantees the efficient DNA delivery to the target organ.

We first searched for SaCas9 PAM sequences (NNGRRT) (28) next to the albumin stop codon, located in exon 14. We identified 5 PAM sequences, 4 upstream (3 in intron 13, and 1 overlapping exon 14 and intron 13 junction) and 1 downstream of the stop codon, in intron 14 (Supplemental Figure 1A; supplemental material available online with this article; https://doi.org/10.1172/jci.insight.128863DS1). The corresponding sgRNAs were cloned into the pX601 vector, a plasmid expressing the $S a C a s 9$ and the sgRNA under the transcriptional control of the CMV and U6 promoters, respectively (Supplemental Figure 1B). To determine their efficacy, we transiently cotransfected HEK293 cells with the different pX601 versions and a homologous recombination (HR) reporter vector (32) containing the albumin exon 14 and flanking introns (Supplemental Figure 1, C and D). The sgRNA7 and sgRNA8 were the most active ones in reconstituting luciferase activity (Supplemental Figure 1E) and were selected for further testing.

Both sgRNA7 and sgRNA8 were able to target the endogenous albumin locus in vitro, in murine NIH-3T3 cells (Figure 2A). Genomic DNA from transfected cells was PCR amplified and analyzed by the T7E1 assay (Figure 2B), resulting in the expected fragments. In parallel, the undigested PCR products were cloned and 48 independent clones were sequenced, revealing the presence of INDELs in 38\% and $71 \%$ of sgRNA7- and sgRNA8-sequenced clones, respectively (Supplemental Figure 2).

We next tested the sgRNA7- and sgRNA8-SaCas9 in vivo in WT newborn mice. We cloned the selected sgRNAs in the pX602 vector, which expresses the SaCas9 under the liver-specific thyroid-binding globulin (TBG) promoter. P4 mice were transduced with single rAAV8 vectors (rAAV-SaCas9-sgRNA7 and rAAV-SaCas9-sgRNA8) (Figure 2C). Determination of targeting efficiency at P19 by the T7E1 assay showed that the sgRNA8 was the most efficient one (Figure 2D), confirming the in vitro results (Supplemental Figure 1E, Supplemental Figure 2, and Figure 2B). Therefore, we selected the sgRNA8 for the next experiments. Importantly, the sgRNA8 target site is located in the intron downstream of the albumin stop codon, at more than 100 bases from the $5^{\prime}$ end of intron 14 (Supplemental Figure 1A). We hypothesized that this characteristic may limit potential risks of damaging the $5^{\prime}$ splice site of albumin exon 14 and, thus, albumin pre-mRNA splicing in targeted alleles not corrected by HDR, because of the generation of large deletions by the error-prone NHEJ DNA repair mechanism. Sequencing of the 
A

SaCas9

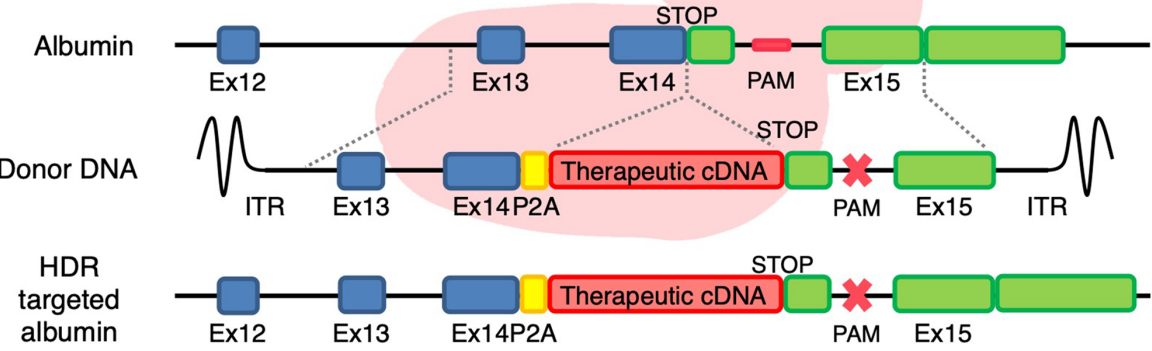

B $\quad$ IAAV-MEDIATED GENOME EDITING

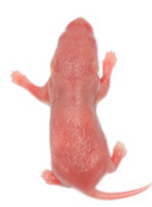

LIVER DYSFUNCTION
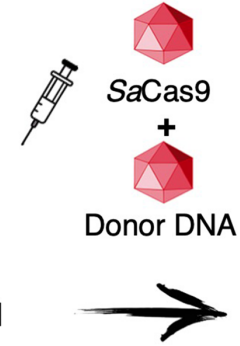

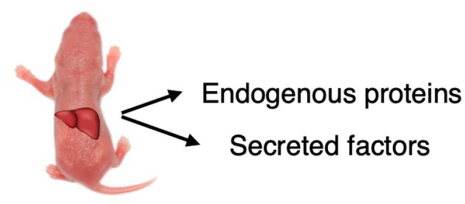

LIVER FUNCTION RESTORATION

Figure 1. General strategy of AAV8-mediated GeneRide in the albumin gene in combination with the CRISPR/SaCas9 platform to correct different liver metabolic diseases. (A) Schematic representation of the experimental strategy. Recombination of the donor vector (Donor DNA; containing the therapeutic cDNA preceded by the 2A-peptide [P2A], and flanked by albumin homology regions, with the PAM8 sequence modified) results in the HDR-targeted albumin allele, a fused mRNA, and translation into 2 separate proteins. Rectangles represent exons; thin black lines, introns; and AAV inverted terminal repeats (ITR), wavy lines. Albumin exons 12 to 15 are indicated; STOP, Albumin stop codon; PAM, protospacer adjacent motif. (B) The therapeutic strategy can be applied to cure different liver metabolic disorders. Neonatal mice with a liver dysfunction are i.v. injected with 2 rAAV8 vectors: one encoding the SaCas9-sgRNA and one the therapeutic donor DNA. The therapeutic cDNA, once integrated, produces the defective or missing protein, such as secreted factors or endogenous enzymes, with the permanent restoration of the liver function.

clones obtained from the PCR products of NIH-3T3 transfected cells (Supplemental Figure 2C) and NGS sequence analysis of the on-target region of DNA extracted from the liver of treated animals (Supplemental Figure 3A) provided experimental support regarding the safety of the procedure. The NGS sequencing mutation analysis showed the precise location of variants in the expected target site, with a median length of the deletions (longer or equal to 2 bases) between 5 and 7 nucleotides, as shown by the gap-length frequency analysis (Supplemental Table 1). The variant frequency analysis showed that the median number of reads with gaps in any nucleotide position surrounding the $\mathrm{SaCas} 9$ cut site was in the range of $3 \%$ to $4.5 \%$, reaching up to $15 \%$ at the $S a C a s 9$ cleavage position, with no signal above noise levels in control samples (Supplemental Figure 3, B and C, and Supplemental Table 2). No significant SNP variants were detected after filtering for sequencing noise.

To set up the most effective conditions, we first performed a dose-finding experiment by i.p. injection of different doses of rAAV-SaCas9-sgRNA8 at P4 in WT mice. The T7E1 assay showed that 7.5E11 viral genomes/mouse (vg/mouse) of rAAV8-SaCas9-sgRNA8 was the lowest dose having the maximum effect (Supplemental Figure 4). We next determined the most effective administration route using an episomal AAV8 vector expressing the EGFP reporter gene under the transcriptional control of a liver-specific promoter (13). i.v. delivery increased the number of EGFP-positive hepatocytes about 8 times (from 0.7 to 5.6 in i.p. and i.v., respectively) (Supplemental Figure 5) and was, therefore, used for the coming experiments.

Efficient targeting of the EGFP cDNA by coupling GeneRide with SaCas9. Next, we tested the previously mentioned selected conditions by coupling GeneRide with $S a C a s 9$ to target an EGFP cDNA into the albumin locus. We i.v.-transduced WT pups with two rAAV8 vectors: one containing the donor DNA with a promoterless EGFP cDNA (rAAV8-donor-EGFP) and one expressing the SaCas9 and the 
A

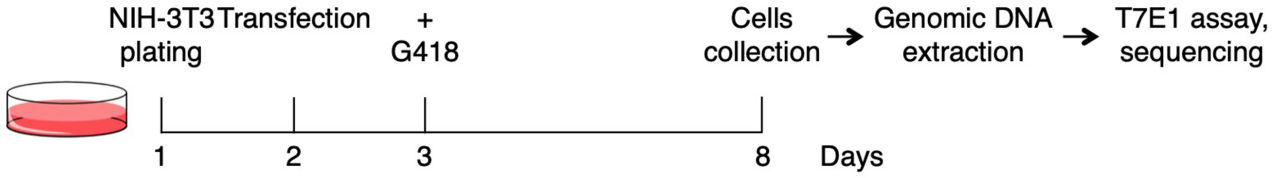

B

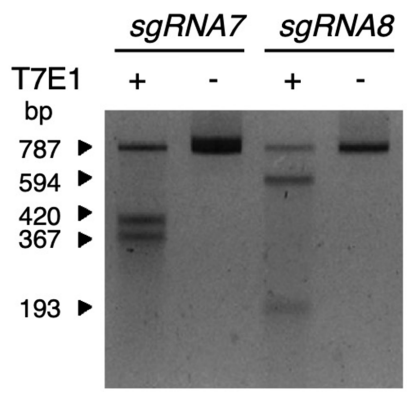

T7E1 ASSAY

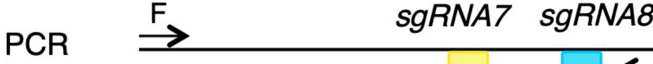
787 bp

+ T7E1

sgRNA7 $420 \mathrm{bp}$

sgRNA8 $594 \mathrm{bp} \quad 193 \mathrm{bp}$

C

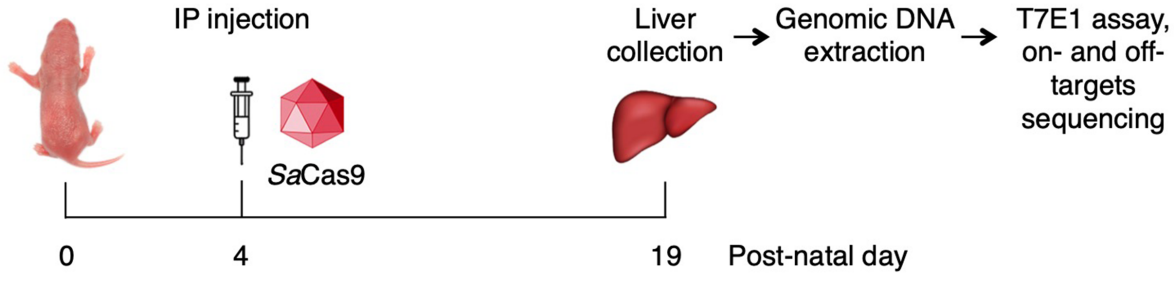

D
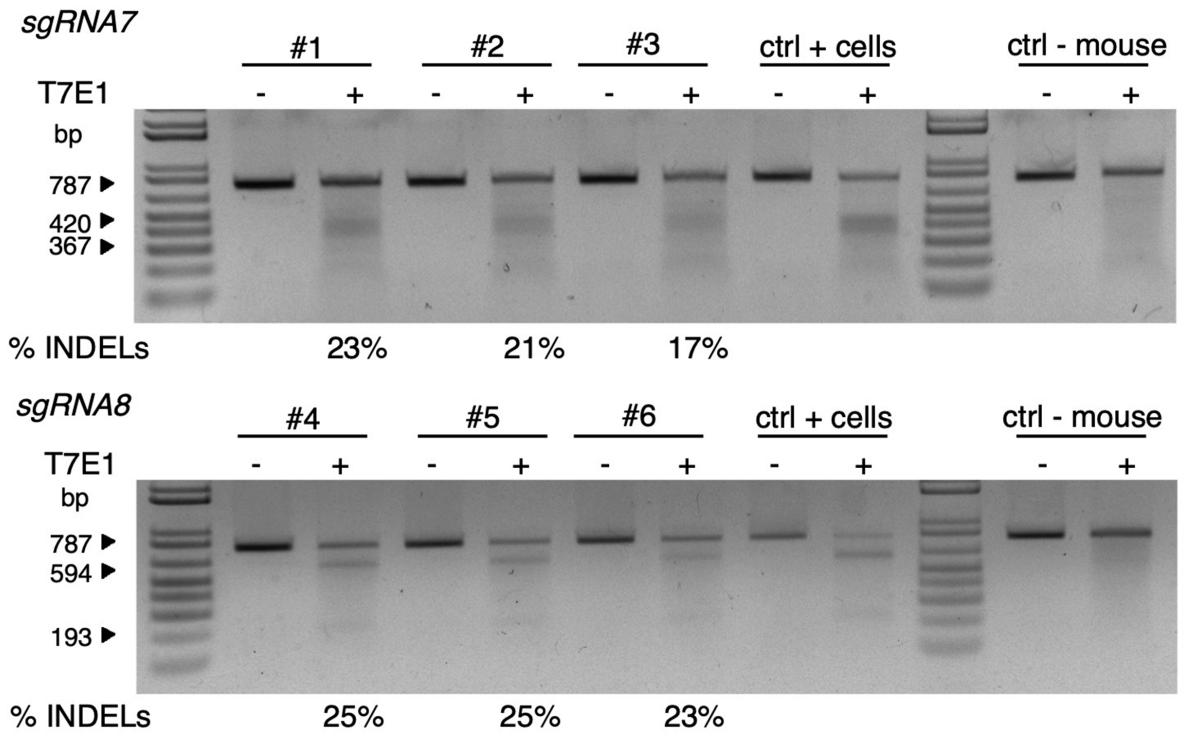

Figure 2. The sgRNA8 is the most active sgRNA in targeting the endogenous albumin locus. (A) In vitro experimental scheme. NIH-3T3 mouse fibroblasts were transfected with the pX601-SaCas9 vectors and collected at day 8, after the addition of G418 (day 3). Genomic DNA was extracted, the target region PCR amplified, and the T7E1 assay and sequencing analysis were performed. (B) The expected bands of T7E1 assay were observed for both sgRNA7 and sgRNA8 transfected cells. T7E1 + or -, treated or not with the T7E1 endonuclease. The size of the fragments is indicated. Right panel: $F$ and R, forward and reverse primers used in the PCR amplifications. The uncut gels are provided in Supplemental Figures 15 and 16. (C) In vivo experimental scheme. WT newborn mice were i.p. transduced at P4 with rAAV8-SaCas9-sgRNA7 or rAAV8-SaCas9-sgRNA8 (1.0E12 vg/mouse) and the liver was collected at P19. Genomic DNA was extracted, the target region PCR amplified, and the T7E1 assay and NGS sequencing were performed. IP, i.p. (D) The T7E1 results for the sgRNA7 and sgRNA8 T7E1 assays are shown. The INDEL \% is indicated. Three mice/sgRNA were treated and analyzed; ctrl + cells, cells treated with the corresponding pX601-SaCas9 vector, ctrl-mouse, untransduced mice. The uncut gels are provided in Supplemental Figures 15 and 16. 
A

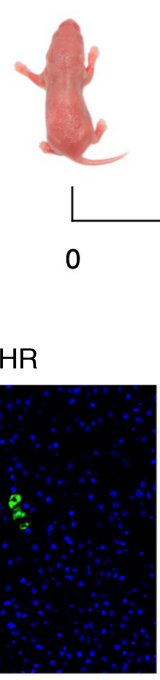

eGFP/Hoechst
IV injection
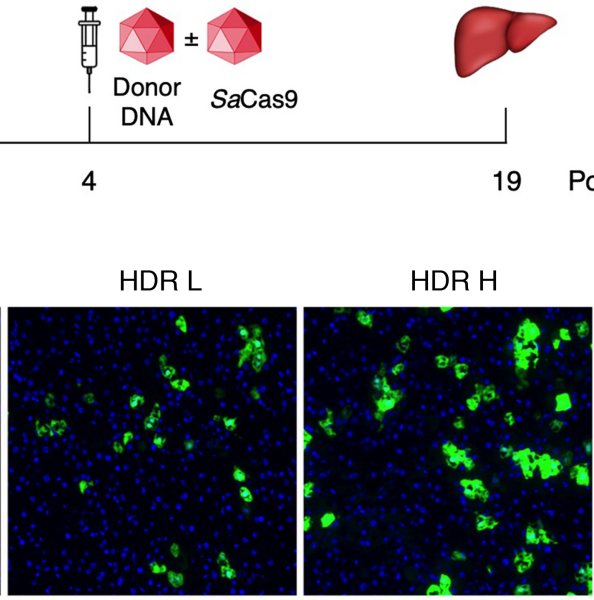

is

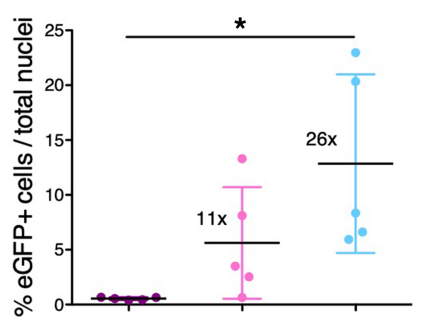

HR HDRL HDRH rAAV8 - Treatment

C
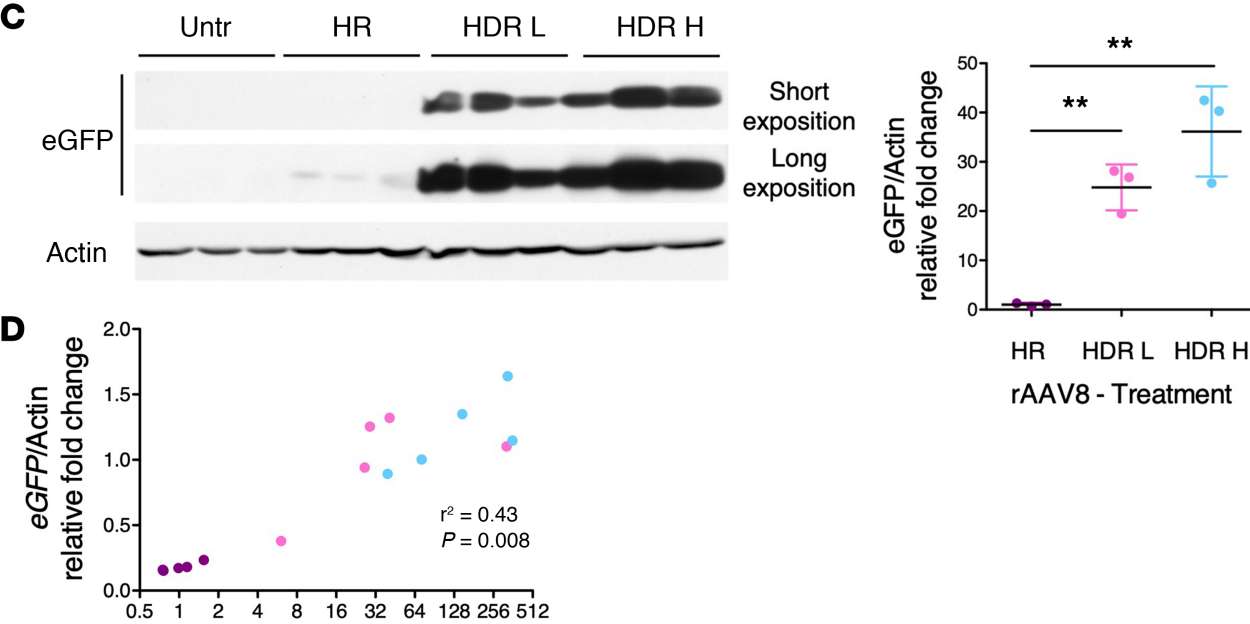

HR HDRL HDR H

rAAV8 - Treatment

Figure 3. Efficient targeting of the ECFP cDNA by coupling GeneRide with SaCas9 in WT newborn mice. (A) Scheme of the experimental design. WT newborn mice were i.v. transduced with the indicated AAV8 vectors. Livers were collected at P19 and analyzed. (B) Histological analysis of liver sections of mice treated with the rAAV8-donor-EGFP alone (HR; 8.0E11 vg/mouse of rAAV8-donor-EGFP) or in combination with rAAV8-SaCas9-sgRNA8, with 2 different SaCas9 doses (low, HDR L; or high, HDR H; $2.0 \mathrm{E} 11$ or $6.0 \mathrm{E} 11 \mathrm{vg} /$ mouse, respectively). Nuclei were counterstained with Hoechst. $n=5$ per group. Scale bar: $500 \mu \mathrm{m}$. One-way ANOVA: ${ }^{*} P=0.0141$; Bonferroni's comparison test: HR versus HDR $L, t=$ 1.447, ns; HR versus HDR $\mathrm{H}, t=3.506, P<0.005$; HDR L versus HDR $\mathrm{H}, t=2.059$, $\mathrm{ns} ; n=5$ per group. (C) WB analysis of liver protein extracts. Short and long expositions are shown. One-way ANOVA: ${ }^{* * *} P=0.0010$; Bonferroni's multiple test: HR versus HDR L, $t=4.914,{ }^{*} P<0.01$; HR versus $\mathrm{HDR} \mathrm{H}, t=7.256,{ }^{*} P<0.01$; $\mathrm{HDR} \mathrm{L}$ versus $\mathrm{HDR} \mathrm{H}, t=2.342$, ns $n=3$ per treatment. The uncut gels are provided in Supplemental Figure 17. (D) Correlation between EGFP and hybrid Alb-EGFP mRNA levels. Correlation, ${ }^{*} P=0.0084, r^{2}=0.4257 ; n=5$ per treatment; 10 images per animal were analyzed.

sgRNA8 (rAAV8-SaCas9-sgRNA8). We tested 2 different donor-SaCas9 ratios, maintaining the dose of the eGFP-donor vector constant and varying the SaCas9-sgRNA8 one (Figure 3A). To determine the increase in recombination rate induced by the generation of DSBs versus spontaneous recombination, we also transduced animals with only the EGFP-donor vector. Potential cleavage of the donor template by the $S a C a s 9$ (as episome or after HDR) was avoided by mutagenizing the sgRNA8 PAM sequence in the donor vector.

Fluorescence microscopy analysis of liver sections showed about $6 \%$ and $13 \%$ of EGFP-positive hepatocytes in the low and high $\mathrm{SaCas} 9$ dose, respectively, with up to $24 \%$ of EGFP-positive hepatocytes and an overall increase of approximately 26-fold with respect to spontaneous HR (Figure 3B). 
Western blot analysis and qRT-PCR confirmed the increase in EGFP protein and mRNA levels, respectively, in the animals transduced with donor DNA and SaCas9-sgRNA8, compared with the group without nuclease. In addition, we observed a good correlation between EGFP protein and mRNA levels in treated mice (Figure 3, C and D).

To further improve the overall efficacy of the approach, we compared the spontaneous recombination rate of the rAAV8-donor-EGFP vector at $\mathrm{P} 2$. We observed that i.v. administration at $\mathrm{P} 2$ resulted in approximately a 3- to 4-fold higher number of EGFP-positive hepatocytes than at P4, reaching approximately $2 \%$ of spontaneous recombination (Supplemental Figure 6); therefore, this condition was selected for the next experiments.

Long-lasting reduction of plasma bilirubin to WT levels in Crigler-Najjar mice. To determine the therapeutic efficacy of the approach, we transduced Crigler-Najjar newborn mice, which present early neonatal lethality $(29,30)$. Because the targeting rate at P2 was 3- to 4-fold more efficient than at P4 (Supplemental Figure 6), we reduced the AAV dose 4-fold, maintaining the donor- $\mathrm{SaCas} 9$ ratio constant. Thus, P2 pups were i.v. injected with the rAAV8 vector encoding the hUGT1A1-donor alone (rAAV-donor-hUGT1A1) or in combination with the rAAV8 vector encoding the $S a C a s 9-s g R N A 8$ (rAAV8-SaCas9-sgRNA8). We tested 2 different donor-SaCas9 ratios, maintaining the dose of the rAAV8-donor-hUGT1A1 vector constant, in combination with the low or high dose of the rAAV-SaCas9-sgRNA8 vector. Mice were temporarily maintained under phototherapy up to P8 (a treatment that does not rescue neonatal lethality) to allow gene targeting and gene expression to occur, avoiding the risk of early bilirubin-induced neurological damage. Animals were sacrificed at 10 months (M10) for analysis (Figure 4A). All rAAV8-treated mice survived, whereas those who received only phototherapy up to P8 died before day 19 (Figure 4B). Mice treated with only the rAAV8-donor- $h U G T 1 A 1$ vector presented life-compatible plasma bilirubin levels that were below toxic levels but still too high for a potential clinical application, confirming previous data (20). Importantly, mice treated with both rAAV vectors had plasma bilirubin levels similar to those of WT littermates for all the duration of the experiment (Figure 4C). Western blot analysis showed that those animals had UGT1A1 protein levels that were about 4- to 6-fold higher than those present in the same amount of WT liver protein extracts (Figure 4D). Importantly, we have not observed any band corresponding to the full-length albumin-P2A-hUGT1A1 protein, suggesting efficient ribosomal skipping by the P2A (expected size $~ 120-125 \mathrm{KDa}$; Supplemental Figure 7). Immunofluorescence analysis of liver sections with a human-specific anti-UGT1A1 antibody revealed that about $3.0 \%-3.6 \%$ of cells were hUGT1A1 positive, with clusters of hUGT1A1-expressing hepatocytes consistent with early recombination events and clonal proliferation of the "targeted" cells (Fig ure 5, A and B). Immunostaining of cerebellar sections showed normal dendritic arborization and tissue architecture (Supplemental Figure 8, A and B), in line with the presence of normal plasma bilirubin levels.

Normal histological and immunological analyses in all treated mice, with no SaCas 9 off-target activity. To determine whether the expression of $\mathrm{SaCas} 9$ and the supraphysiological levels of hUGT1A1 may have undesired consequences in animal health, we performed histological analysis of liver sections. H\&E and Masson's trichrome stainings showed normal histology in all treated and untreated mice, with no signs of liver fibrosis or infiltration (Supplemental Figure 9). mRNA analysis of inflammatory markers by RT-qPCR showed no significant differences among all analyzed groups (Supplemental Figure 10), confirming the normal condition of the livers of all animals. Importantly, to assess whether the treatment may affect albumin expression levels, we determined albumin levels in plasma of WT mice treated at P2 with EGFP-donor DNA alone (HR), or in combination with $\mathrm{SaCas9-encoding} \mathrm{vector,} \mathrm{at} \mathrm{low} \mathrm{and} \mathrm{high}$ SaCas9 doses (HDR L and HDR H, respectively; Supplemental Figure 11, A and B). We also determined albumin mRNA levels in WT mice treated only with rAAV8-SaCas9-sgRNA8, and in $\mathrm{Ugt}^{1^{-/-}}$mice treated with both rAAV8-SaCas9-sgRNA8 and rAAV8-donor-hUGT1A1 vectors (Supplemental Figure 11, C and D). We observed that neither plasma albumin nor albumin mRNA levels were affected by the treatments at any of the time points and conditions analyzed.

One important concern when delivering SaCas9 with AAV is the potential long-term expression of the nuclease in the target tissue, which may lead to increased off-target activity and genotoxicity. Therefore, we analyzed SaCas9 endonuclease levels by Western blot at different time points after AAV delivery. Western blot analysis showed that liver protein levels of $\mathrm{SaCas} 9$ waned to undetectable levels at P30 (Supplemental Figures 12 and 13), most likely the consequence of vector loss associated to hepatocyte duplication during liver growth (12-14).

To assess the risks of nuclease off-target activity, we first performed an in silico prediction analysis of potential off-target sites with the bioinformatics software Cas-OFFinder (http://www.rgenome.net/cas-offinder). We found no off-target sites having less than 4 nucleotides mismatches, whereas only 4 potential off-target sites were 
A

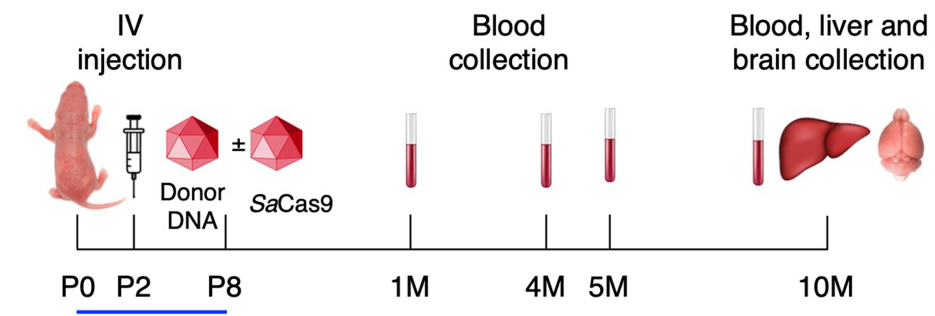

B

PT Days of age $(P)$

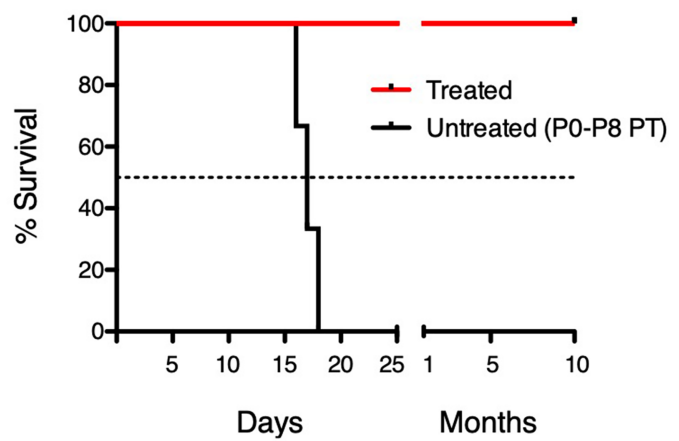

C Months of age (M)

D
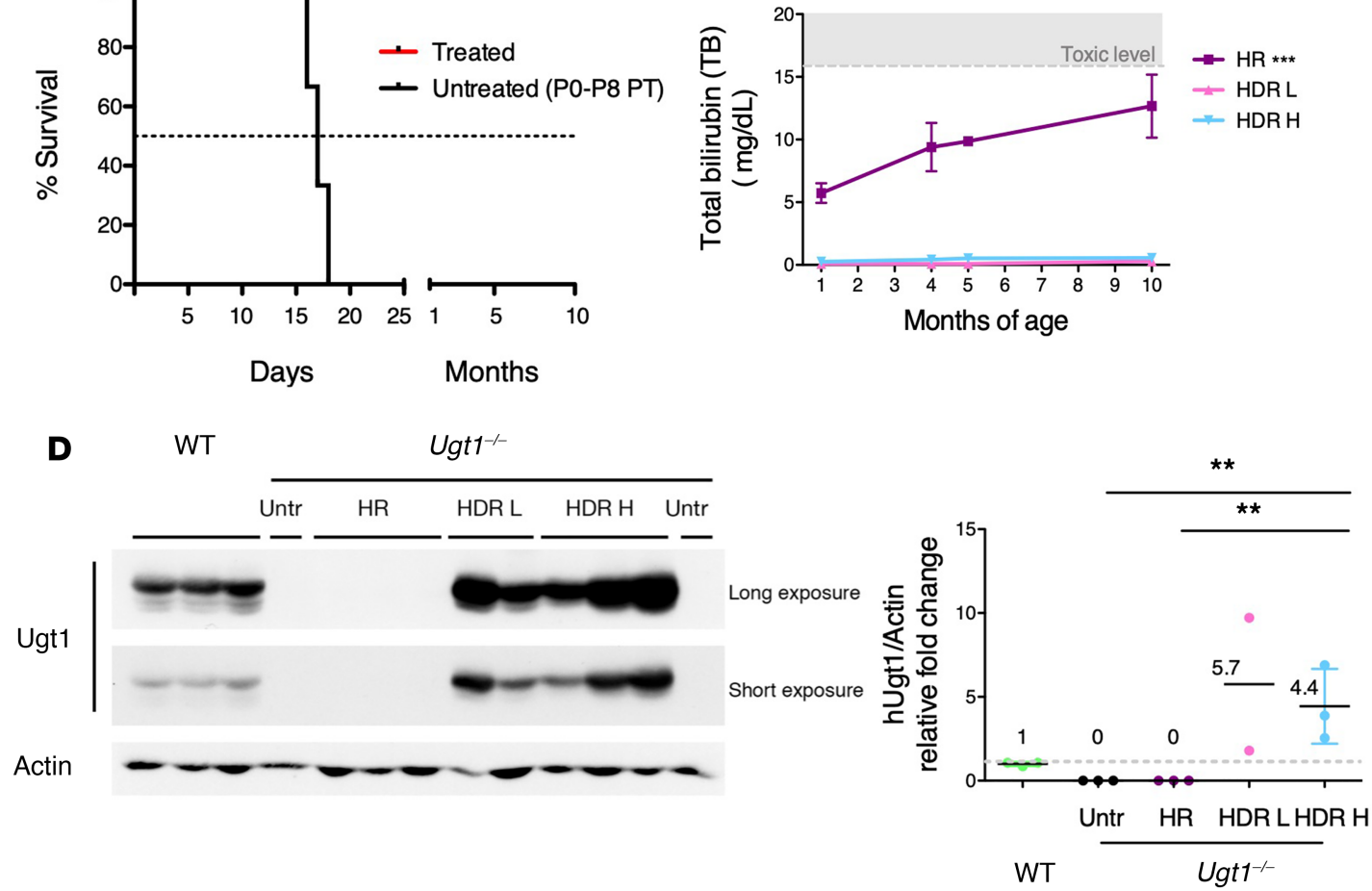

Figure 4. Long-lasting reduction of plasma bilirubin to $\boldsymbol{W} \boldsymbol{T}$ levels in Crigler-Najjar mice. (A) Scheme of the experimental design. Ugt1 ${ }^{-/-}$newborn mice were i.v. transduced at P2 with rAAV8-donor-hUGT1A1 alone (HR; $2.0 \mathrm{E} 11 \mathrm{vg} / \mathrm{mouse}$ ) or in combination with rAAV8-SaCas9-sgRNA8, using 2 different SaCas9 doses (low, HDR L; or high, HDR H; 6.0E10 and 2.0E11 vg/mouse, respectively). Mice were maintained under phototherapy (PT) from birth to P8 (P0-P8 PT). Blood was collected at the indicated time points and mice were sacrificed at M10. Liver and brain were extracted. (B) Kaplan-Meier survival curve. All rAAV8-treated mice survived, whereas all mutant mice treated only with PT up to P8 $(n=3)$ died before P19. Long-rank (Mantel-Cox) test, ${ }^{* *} P=$ 0.0020. $n=3$ per rAAV8-untreated, $n=8$ per rAAV8 treated. (C) Total bilirubin (TB) levels were determined in plasma at 1, 4, 5, and 10 months. TB levels of mice treated with both rAAV8 vectors (HDR L and HDR H) were similar to WT/HET. The gray area in the graph indicates the range of TB levels resulting in brain damage and death. Two-way ANOVA: interaction, ${ }^{* * *} P=0.0010$; treatment, ${ }^{* * *} P<0.0001$; time, ${ }^{* *} P=0.0015$. Bonferroni post-test, HR versus HDR $\mathrm{L} / \mathrm{HDR} \mathrm{H},{ }^{* * *} . n=3$ per HR and HDR H; $n=2$ per HDR L. The uncut gels are provided in Supplemental Figure 7. (D) WB analysis of liver protein extracts using an anti-Ugt1 antibody with human and mouse specificity. Short and long expositions are shown. Quantification of the WB. One-way ANOVA, ${ }^{* *} P=0.0036$. Bonferroni's multiple comparison test: Untr vs. HDR H; ${ }^{* *}, \mathrm{HR}$ vs. HDR H, ${ }^{* *} . n=3$ per WT and HDR $\mathrm{H}, n=2$ per Untr, HDR L. ND, not detected. ${ }^{* *} P<0.01$.

identified with 4 mismatches. Because it was previously shown that $\mathrm{SaCas} 9$ INDELs were not present in sites containing 5 or more mismatches (28), we further investigated only those with 4 mismatches. Based on genome location within actively transcribed regions and their functional importance, we selected Tubgcp2, Kif21a, and Gm29874 genes. Although Tubgcp2 and Kif21a are protein-coding genes, Gm29874 is, instead, a long noncoding RNA gene. The fourth potential off-target locus ( $N m f 420$ ) was not further analyzed because it corresponds to an intergenic, noncoding region, with unknown function. Then, the identified genomic regions were PCR amplified from rAAV-SaCas9-sgRNA8-treated livers and deep sequenced. Conservative filtering was applied to reduce the number of false negatives as much as possible. We did not observe the presence of INDELs or SNPs in the samples from either treated or untreated animals above background levels (Supplemental Figure 14 and Supplemental Tables 3 and 4), providing key experimental support to the safety of the procedure. 
A
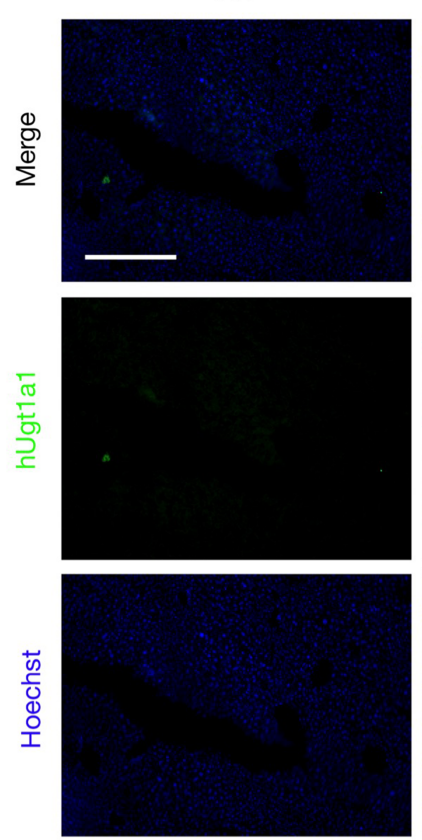

B

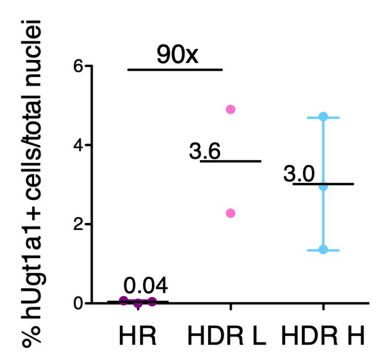

HDR L
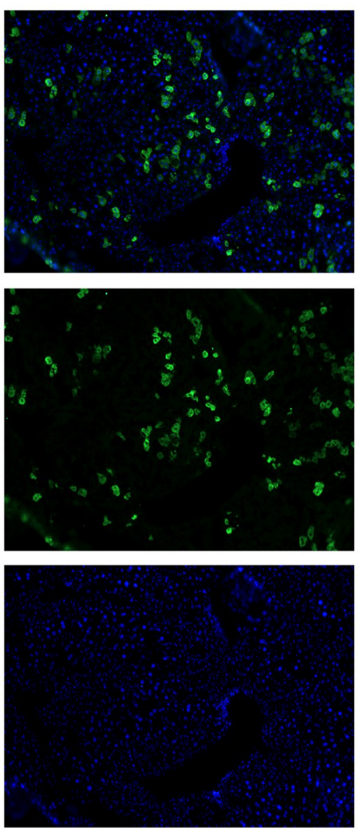

HDR H
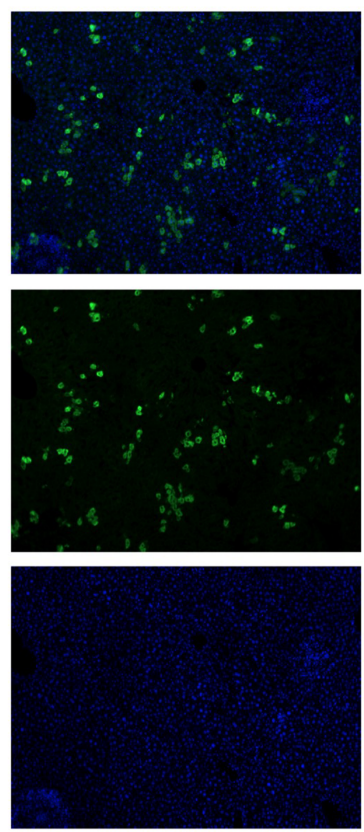

Figure 5. Immunofluorescence analysis of liver sections shows a high number of recombinant hepatocytes in rAAV-treated $\mathrm{Ugt1}^{-/-}$mice. (A) Immunofluorescence analysis of liver sections of $\mathrm{Ugt1}^{-1-}$ mice treated with rAAV8-donor-hUGT1A1 alone (HR) or in combination with rAAV8-SaCas9-sgRNA8, using 2 different doses (HDR L and HDR H, for low and high rAAV8-SaCas9-sgRNA8 vector doses, respectively), as described in Figure 4. Sections were stained with a human-specific anti-UGT1A1 antibody. Nuclei were counterstained with Hoechst. $n=3$ per HR and HDR H; $n=2$ per HDR L. (B) Quantification of hUGT1A1 positive hepatocytes of liver sections. Student's $t$ test, HR vs. HDR H, ${ }^{*} P=$ 0.0372. HDR L, $n=2$; HR, HDR H, $n=3$.

\section{Discussion}

An attractive possibility to cure liver diseases with neonatal/pediatric onset is the permanent modification of the genome, by which the targeted allele is stably transmitted to daughter cells during proliferation. Recently, CRISPR/Cas9-mediated gene-editing approaches for disease-causing mutations have been successfully applied to mouse models of hereditary tyrosinemia type I (HTI) (24) and ornithine transcarbamylase deficiency (OTCD) (26), demonstrating their feasibility. However, the transfer of these strategies to the clinic is hampered by the presence of multiple disease-causing mutations, as exemplified in 2 of the most paradigmatic liver metabolic diseases, the $\operatorname{CNSI}(33,34)$ and $\operatorname{OTCD}(35,36)$, implying that several mutation-specific gene editing strategies and vectors are required to cure these diseases.

These concerns can be overcome by the integration of a therapeutic cassette into a preselected "safe-harbor" locus (37), ensuring long-lasting, robust, and predictable expression with a single therapeutic strategy. We have recently developed a gene-targeting approach (namely "GeneRide") without nucleases based on the in-frame insertion of a promoterless therapeutic cDNA just upstream of the albumin stop codon (19). This strategy resulted in the amelioration of the bleeding diathesis in hemophilia B mice (19) and the rescue of a lethal mouse model of the CNSI (20). Here, we further increased the recombination rate and therapeutic efficacy by combining GeneRide with the CRISPR/SaCas9 platform. The proposed approach has several advantages over existing methods: (a) higher gene-targeting rate; (b) higher levels of transgene 
expression; (c) permanent modification of the genome with life-long therapeutic efficacy; (d) albumin gene expression is not affected; (e) the same strategy and AAV vectors can be applied to most disease-causing mutations; (f) different metabolic disorders can be treated by just replacing the therapeutic cDNA in the donor construct; and ( $\mathrm{g}$ ) high specificity of the approach with no off-target activity in predicted sites and no detected tumorigenesis.

The CNSI mice closely mimic the main features of the human syndrome, with neonatal severe unconjugated hyperbilirubinemia and early neonatal lethality $(29,30,38)$. Although nonintegrative AAV-mediated gene therapy is very effective in rescuing the phenotype in adult CNSI animals $(39,40)$, it requires high doses of liver-specific rAAV8 episomal vectors expressing the UGT1A1 transgene when administered to newborn mutant mice $(13,41,42)$. However, therapeutic efficacy decreases over time and a second administration is necessary to achieve full correction in the long term $(41,42)$. Similarly, neonatal AAV delivery also results in loss of therapeutic efficacy in other liver diseases, such as OTCD (12), underlining the limits of nonintegrative AAV-mediated gene therapy for severe liver disorders with neonatal and pediatric onset. In the present work, we demonstrated that GeneRide coupled to CRISPR/SaCas9 platform resulted in a substantial improvement of the gene-targeting rate in vivo by fully rescuing a lethal mouse model of the CNSI, with normal cerebellar architecture and plasma bilirubin levels similar to WT for the whole duration of the experiment (10 months). This approach permanently modifies the hepatocyte genomes and, in line with previous results $(19,20)$, recombinant cells were found in small clusters providing strong evidence that the inserted transgene is stably transmitted to daughter cells during hepatocyte proliferation, also suggesting that recombination is an early event that occurs upon AAV transduction.

An additional strength of the proposed strategy resides in the capability to correct most existing mutations of a given diseased gene with the same therapeutic rAAV tools. Furthermore, the high versatility and potentiality of the approach are demonstrated by the possibility to use the same AAV vector carrying the $S a$ Cas 9 and the sgRNA to correct different metabolic liver diseases. It is necessary only to replace the therapeutic cDNA in the donor $\mathrm{AAAV}$ vector that will be targeted into the albumin locus, remaining under the transcriptional control of the robust albumin promoter and guaranteeing life-long high levels of transgene expression.

The data obtained showed a much higher efficacy than other gene-targeting approaches using nucleases. In fact, we observed $13 \%$ and $3 \%-4 \%$ of recombinant hepatocytes with the EGFP and hUGT1A1 transgenes, respectively. Sharma et al., instead, targeted the first albumin intron obtaining values in the range of $0.5 \%$ (43). The differences in the gene-targeting rate between the 2 experimental approaches may be related to the targeted region and arms of homology, nucleases, transgenes, age of the animals, or a combination of these factors. In fact, we also observed differences in the gene-targeting rate between the EGFP and $h U G T 1 A 1$ donor vectors, which may be related to the different cDNA length. Given the apparent higher recombination rate of the strategy proposed here, if applied into a clinic setting it may require lower rAAV doses, with an important increase in both therapeutic efficacy and safety.

The therapeutic level required to correct the diseased phenotype varies among the different liver disorders, ranging from $2 \%$ to $5 \%$ of functional protein to provide therapeutic benefits in hemophilia (44), $5 \%$ to $10 \%$ of enzyme activity for CNSI $(13,38,45,46)$, or even higher levels in the case of urea cycle disorders (47). Our approach resulted in long-term therapeutic levels of the UGT1A1 enzyme, with protein levels higher than those of WT animals. Thus, it is expected that the approach will also be effective for hemophilia, urea cycle disorders, and probably other liver diseases. This methodology is particularly attractive for diseases with neonatal or pediatric onset, a period characterized by hepatocyte duplication during liver growth, because proliferating cells present a higher HDR rate (48) and nonintegrative gene therapy is not effective (12-14). Although previous experiments showed no significant differences in spontaneous gene-targeting rate between neonatal and adult delivery in mice (19), the HDR rate may differ in the presence of DSBs generated by nucleases and further experiments may be required to determine the potential application of the approach to adults. In fact, most adult hepatocytes are quiescent and do not duplicate, being DSBs mainly repaired by NHEJ, as observed after CRISPR/SaCas9-mediated editing of adult $O T C^{\text {Spf-Ash }}$ mice (26). In addition, we have also observed differences in the gene-targeting rate between donor DNAs containing the EGFP or the hUGT1A1 cDNAs, both in the presence or absence of nucleases (this paper and ref. 20). Thus, the effective gene-targeting rate should be experimentally determined for each disease model. 
The use of engineered endonucleases raises important concerns related to the long-term presence of the nuclease in the targeted cells or tissues, immunogenicity, potential off-target activity, and risk of tumorigenesis. The $S a C a s 9$ nuclease has a cleavage efficiency similar to that of the more popular $S p$ Cas 9 (28), and one of its major advantages is its smaller size, allowing its packaging into AAV vectors along with the sgRNA (49). In addition, the SaCas9 PAM sequence is more complex, resulting in a lower number of potential off-target sites (28). In silico prediction and molecular analysis of potential off-target sites showed no detectable off-target activity in the selected sites analyzed. In addition, we have not observed any adverse effect consequent to the treatment or to the presence of supraphysiological levels of the therapeutic protein, such as induction of tumorigenesis, liver inflammation, or changes in plasma albumin levels. However, more extensive characterization of these potential undesired effects and the potential long-term presence of the nuclease in the target tissue is required to consider the translation of this approach to a clinical setting. Different methodologies have been proposed to reduce the potential risks of off-target activity, such as using high-fidelity Cas9 nucleases (50-52), reducing the time window in which the nuclease is present in the target tissue with self-limiting Cas9 circuits, or delivering the endonuclease as mRNA or protein using lipid nanoparticles $(25,53-57)$. An additional concern of the approach is the potential inactivation of the albumin gene by NHEJ repair of the DSBs. Molecular analysis of the on-target site showed that most gaps were shorter than 7 nucleotides, and we have not observed changes in plasma albumin levels, supporting the safety of the approach. We have seen, however, that a very small fraction of the DSBs in the albumin gene, not repaired by HDR, resulted in large deletions generated by NHEJ-mediated repair, as observed for other on-target loci in mouse ES cells and bone marrow cells (58).

The proposed platform provides proof-of-concept evidence for long-lasting efficacy and safety in a lethal mouse model of the CNSI. Further studies will be required to demonstrate its therapeutic value in other liver metabolic diseases and to further improve its safety profile before its potential translation into the clinic.

\section{Methods}

\section{Animals}

Animals were housed and handled according to institutional guidelines. Ugt $1^{-1-}$ mice used were at least 99.8\% FVB/NJ genetic background (29), obtained after more than 10 backcrosses with FVB/NJ WT mice. Males and females were used indistinctly for the experiments. Mice were maintained in the ICGEB animal house unit in a temperature-controlled environment with 12-hour light/dark cycles and received a standard chow diet and water ad libitum.

\section{Animal treatments}

Phototherapy treatment. Ugt $1^{-1-}$ and WT newborn littermates were exposed to blue fluorescent light as previously described (30) up to P8 and then maintained under normal light conditions. This sole treatment

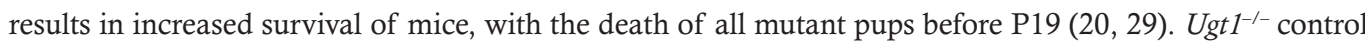
mice were obtained by treating mutant mice with phototherapy for 15 days after birth. This temporary treatment results in survival of all mice after discontinuation of the phototherapy treatment (29).

$A A V$ treatments. AAV-treated mice at a different postnatal day (P4 or P2) were i.p. or i.v. injected as described previously $(30,39,40)$, with the indicated vectors, at the indicated dose, as described in Supplemental Table 5. Briefly, for EGFP editing experiments, WT mice were i.v. injected at P4 with rAAV8-donor-EGFP (8.0E11 vg/mouse; HR, HDR L, HDR H) and rAAV8-SaCas9-sgRNA8 (2.0E11 or 6.0E11 vg/ mouse, HDR L, HDR H). For hUGT1A1 editing experiments, Ugt $1^{-1-}$ mice were i.v. injected at P2 with rAAV8-donor-hUGT1A1 (2.0E11 vg/mouse; HR, HDR L, HDR H) and rAAV8-SaCas9-sgRNA8 (6.0E10 or 2.0E11 vg/mouse, HDR L, HDR H).

\section{Construction of sgRNAs-SaCas9 and donor DNA AAV vectors}

sgRNAs were designed based on the $S a C a s 9$ PAM sequences (NNGRRT) identified next to the albumin stop codon. sgRNA coding oligonucleotides, specified in Supplemental Table 6, were cloned into pX601 and pX602 plasmids, obtained from Addgene (http://www.addgene.org). Both plasmids encode for SaCas 9 and for an incomplete sgRNA sequence. In the pX601 and pX602 plasmids, the SaCas9 is under the transcriptional control of the cytomegalovirus (CMV) or thyroid-binding protein (TBG) promoters, respectively. All identified 
sgRNAs were cloned into the pX601 plasmid, whereas only sgRNA7 and sgRNA8 were further cloned into pX602 for the production of $\mathrm{rAAV}$ vectors for the in vivo experiments.

The EGFP- and $h U G T 1 A 1$ - donor vectors (donor-EGFP and - $h U G T 1 A 1$ ) were prepared as previously described (20), but the PAM sequence of the sgRNA8 was mutated to avoid targeting to the donor vector. As previously done for $h F I X(19)$, to reduce the risk of potential off-target expression, our vectors have no ATG start translation codon neither in the 2A-peptide, nor in the EGFP and hUGT1A1 cDNAs. The NcoI-PflMI fragment of the original donor vector (20) was replaced with a synthesized fragment containing the PAM8 modified. For episomal expression, an AAV8-AAT-EGFP vector was used (13).

\section{Cell transfections}

HEK293 cells were transfected with the pX601 plasmids encoding for the 5 different sgRNAs and the SaCas9, together with a HR reporter vector (32), using Lipofectamine 2000 (Invitrogen) following the manufacturer's guidelines. The reporter vector was modified by replacing the sequence of the UGT1 Exon 4 with a fragment of the albumin gene containing all sgRNAs target sequences.

NIH-3T3 mouse fibroblasts were transfected with pX601 plasmids encoding sgRNA7 or sgRNA8 and pEGFP-C2 plasmid using Lipofectamine LTX (Invitrogen), following manufacturer's instructions.

\section{Genomic DNA preparation from cells and from liver samples}

Genomic DNA was prepared from cells as briefly described below. Cells were lysed in $50 \mathrm{mM}$ Tris- $\mathrm{HCl} \mathrm{pH}$ 7.5, $100 \mathrm{mM}$ EDTA, $0.5 \% \mathrm{SDS}$, and $200 \mu \mathrm{g} / \mathrm{mL}$ protease $\mathrm{K}$ for 1 hour at $37^{\circ} \mathrm{C}$ and centrifuged for $10 \mathrm{~min}$ utes at maximum speed. Genomic DNA was precipitated with 2-propanol and resuspended in TE buffer after centrifugation and washing.

Whole livers were extracted and reduced to powder with a mortar and liquid nitrogen and stored at $-80^{\circ} \mathrm{C}$. Genomic DNA was prepared incubating as previously described (41).

\section{T7E1 assay}

The T7E1 assay was performed following the manufacturer's instructions (New England Biolabs). PCR reactions were performed using the oligonucleotides specified in Supplemental Table 7, according to the protocol of the Taq polymerase used (Roche). The PCR program was optimized with the following cycles: 1 cycle of 2 minutes at $94^{\circ} \mathrm{C} ; 30$ cycles of 30 seconds at $94^{\circ} \mathrm{C}, 45$ seconds at $60^{\circ} \mathrm{C}$ and 1 minute at $68^{\circ} \mathrm{C}$; and 1 cycle of 7 minutes at $68^{\circ} \mathrm{C}$. The PCR amplicons were purified with Sephacryl S-400 beads (GE Healthcare Biosciences $\mathrm{AB})$, following the manufacturer's instructions. Purified amplicons were denatured, self-annealed, and treated with the T7E1 enzyme. Digested and undigested mixes were visualized on a $2 \%$ agarose gel. The estimated gene modifications were calculated as described previously (59).

\section{Production, purification, and characterization of the rAAV vectors}

The AAV vectors used in this study are based on AAV type 2 backbone, and infectious vectors were prepared by the AAV Vector Unit at ICGEB Trieste (https://www.icgeb.org/avu-core-facility.html) in HEK293 cells by a cross-packing approach whereby the vector was packaged into AAV capsid 8, as described previously (13).

\section{Bilirubin and albumin determination in plasma}

Blood was obtained from anesthetized mice by facial vein bleeding. Bilirubin and albumin were determined in plasma as described previously $(29,60)$.

\section{RNA preparation and mRNA quantification analysis (qRT-PCR)}

Total RNA from mouse liver powder was prepared using TRI reagent solution (Invitrogen), according to the manufacturer's instructions. cDNA and PCR were done as previously described (41). One $\mu \mathrm{L}$ of cDNA diluted 1:10 was used to perform qPCR using the specific primers listed in Supplemental Table 8 to amplify the hybrid Alb-EGFP RNA, the endogenous mouse albumin cDNA, the inflammatory markers Tnfa, $C d 8$, $C d 4$, and Infg cDNAs, or the Gapdh housekeeping gene. qPCR was performed using the iQ SYBER Green Supermix (Bio-Rad) and a C1000 Thermal Cycler CFX96 Real Time System (Bio-Rad). Data were analyzed using the $\Delta \Delta \mathrm{Ct}$ method.

Expression of the gene of interest was normalized to albumin to estimate the relative expression of the Alb-EGFP cDNA to Gapdh for quantification of the inflammatory markers and albumin expression. 
Total protein extract preparation and Western blot analysis

Liver total protein extracts were obtained as described previously (30). Total protein concentration was determined by Bradford (Bio-Rad). For the EGFP and SaCas9 Western blot analysis, 20 and $40 \mu \mathrm{g}$ of total protein extracts from the liver of WT untreated and treated mice were analyzed, respectively. For the hUGT1A1 Western blot analysis, $40 \mu \mathrm{g}$ of total protein extracts from the livers of WT and mutant untreated and treated mice were analyzed. Primary and secondary antibodies were used as specified in Supplemental Table 9.

\section{Histological analysis}

Liver biopsies of treated animals were fixed as described previously (29). Paraffin-embedded liver sections (5 $\mu \mathrm{m})$ were stained with H\&E and Masson's trichrome as previously described $(29,30)$. For the EGFP, hUGT1A1, and calbindin experiments and immunofluorescence, procedures were performed as described previously $(13,20,29,30)$. Quantification of EGFP-positive cells was performed as follows. Two liver sections per animal, five images per section, were analyzed at $\times 20$ magnification. The ratio between the number of the total EGFP-positive cells and the nuclei per mice was calculated.

Quantification of hUGT1A1-positive cells was performed as follows. Four liver sections per animal were analyzed at $\times 10$ magnification. The ratio of the total number of hUGT1A1-positive cells/total number of nuclei counted in all acquired images per animal was calculated. Measurements were averaged for each animal and the results were expressed as mean \pm SD for each treatment.

Analysis of the layer thickness was performed on Hoechst-stained sections by measuring the layer depth $(\mu \mathrm{m})$ as previously described (30). PC density analyses were performed as previously described $(29,30)$.

Images were acquired on a fluorescence microscopy. Digital images were collected using Leica software and analyzed using ImageJ (NIH).

\section{Sanger sequencing on target in vitro}

Amplicons from sgRNA-treated cells were cloned in pGEM-T vector (Promega); plasmid DNA was purified from positive clones and sent for Sanger sequencing.

\section{NCS sequencing on- and off-targets}

Off-target loci were predicted using the CasOffinder software (http://www.rgenome.net/cas-offinder) (61). Genomic DNA from the predicted off-target regions and that of the albumin on-target site were PCR amplified and sent to BMR Genomics for Illumina sequencing. The primers used for the PCR amplifications are listed in Supplemental Table 10 and contained the complementary regions and a tail on the $5^{\prime}$ and $3^{\prime}$ end, which was necessary for the sequencing reaction. The length of the PCR fragment for the on-target albumin amplicon was $517 \mathrm{bp}$; for the off-target amplicons the length of the PCR fragment was as follows: Tubgcp2, $522 \mathrm{bp}$; Kif21A, $478 \mathrm{bp}$; and Gm29874, $491 \mathrm{bp}$.

Sequenced libraries were first split into locus-specific bins based on the best match to a corresponding source locus using the BBSplit tool (BBtools, https://jgi.doe.gov/data-and-tools/bbtools/) and reads in each bin were counted to verify that they were sufficient to provide deep coverage. Sequencing runs for the Gm29874 locus were subsequently repeated because they were not originally represented in quantities comparable with the other two off-target loci and an on-target locus. Each read bin was then separately mapped to its corresponding locus target with bwa (62). All unmapped reads and reads without both pairs mapping to the target sequence were filtered out with samtools (63). Indels were left-aligned using the GATK LeftAlignIndels function (64). All reads below the mapping quality of Q30 ad positions below the base-calling quality of Q20 were removed from subsequent analysis. Base and indel frequencies were called at each target position from the aligned and post-processed BAM files by using the custom R script based on the Bioconductor's Rsamtools package (Rsamtools, http://bioconductor.org/packages/release/ bioc/html/Rsamtools.html). Samples and targets without the SaCas9 treatment were used as a control and alternative base and indel frequencies (thereinafter jointly termed as alternative variants) were used to model the experimental error with the beta-binomial distribution with the $\mathrm{R}$ ebbr package. Parameters for the beta-binomial function were estimated from all alternative variant count/depth ratios in nontreated samples. The 99.9th percentile of the distribution was taken as the error likelihood threshold and then each sample was statistically evaluated at each position to estimate whether the alternative variant/depth at a specific site within the sample was above or below the likelihood threshold for error. The estimates were done separately for SNPs and indels. Subsequently, each sample's indel and SNP frequency profiles were 
separated into "signal" and "noise" parts, based on whether they exceed the error likelihood. From these profiles, the overall statistics was compiled on the magnitude and overall event count (event = alternative variant in the significant signal range).

\section{Statistics}

The Prism package (GraphPad Software) was used to analyze the data. Results are expressed as mean \pm SD. $P<0.05$ was considered statistically significant. Depending on the experimental design, 2-tailed Student's $t$ test, or 1-way or 2-way ANOVA, with Bonferroni's post hoc comparison tests, was used, as indicated in the legends to the figures and text.

\section{Study approval}

All animal experimental procedures were approved by the ICGEB review board, with full respect to the EU Directive 2010/63/EU for animal experimentation, and by the Italian Health Minister (authorization 996/2017-PR).

\section{Author contributions}

ADC performed most of the experiments and data analysis, and generated figures for the manuscript. FP, GB, RS, and ML helped in the experiments, contributed to the interpretation of results, and provided critical insights into the significance of the work. ADC and GB performed histological staining of liver samples. KV performed the bioinformatics analysis of the SaCas9 on- and off-target activities. AB and MAK provided the original GeneRide construct and important contributions in the design of the experiments. MG and LZ provided important contributions in the experimental design and produced the AAV vectors used in the studies. AFM designed and analyzed the experiments and wrote the manuscript. All authors read and corrected the manuscript.

\section{Acknowledgments}

To Serena Zacchigna for help in the initial stages of virus administration to pups; to the BioExperimentation Facility for help with animal care. This work was supported by intramural funds (to AFM); it was also supported by the NIH R01-HL 064274 (to MAK).

Address correspondence to: Andrés F. Muro, ICGEB, Padriciano, 99, 34149 Trieste, Italy. Phone: 30.040.3757312; Email: muro@icgeb.org.

RS's present address is: Department of Life Sciences, University of Trieste, Trieste, Italy.

1. Fagiuoli S, Daina E, D'Antiga L, Colledan M, Remuzzi G. Monogenic diseases that can be cured by liver transplantation. J Hepatol. 2013;59(3):595-612.

2. Hansen K, Horslen S. Metabolic liver disease in children. Liver Transpl. 2008;14(4):391-411.

3. Adam R, et al. Evolution of indications and results of liver transplantation in Europe. A report from the European Liver Transplant Registry (ELTR). J Hepatol. 2012;57(3):675-688.

4. Herrero JI. De novo malignancies following liver transplantation: impact and recommendations. Liver Transpl. 2009;15(suppl 2):S90-S94.

5. Vajdic CM, van Leeuwen MT. Cancer incidence and risk factors after solid organ transplantation. Int J Cancer. 2009;125(8):1747-1754.

6. Haagsma EB, et al. Increased cancer risk after liver transplantation: a population-based study. J Hepatol. 2001;34(1):84-91.

7. Benten D, Staufer K, Sterneck M. Orthotopic liver transplantation and what to do during follow-up: recommendations for the practitioner. Nat Clin Pract Gastroenterol Hepatol. 2009;6(1):23-36.

8. Nathwani AC, et al. Long-term safety and efficacy of factor IX gene therapy in hemophilia B. N Engl J Med. 2014;371(21):1994-2004.

9. Nathwani AC, et al. Long-term safety and efficacy following systemic administration of a self-complementary AAV vector encoding human FIX pseudotyped with serotype 5 and 8 capsid proteins. Mol Ther. 2011;19(5):876-885.

10. George LA, et al. Hemophilia B Gene Therapy with a high-specific-activity factor IX variant. N Engl J Med. 2017;377(23):2215-2227.

11. Rangarajan S, et al. AAV5-factor VIII gene transfer in severe hemophilia A. N Engl J Med. 2017;377(26):2519-2530.

12. Cunningham SC, Spinoulas A, Carpenter KH, Wilcken B, Kuchel PW, Alexander IE. AAV2/8-mediated correction of OTC deficiency is robust in adult but not neonatal Spf(ash) mice. Mol Ther. 2009;17(8):1340-1346.

13. Bortolussi G, et al. Life-long correction of hyperbilirubinemia with a neonatal liver-specific AAV-mediated gene transfer in a lethal mouse model of Crigler-Najjar Syndrome. Hum Gene Ther. 2014;25(9):844-855.

14. Wang L, Wang H, Bell P, McMenamin D, Wilson JM. Hepatic gene transfer in neonatal mice by adeno-associated virus serotype 8 vector. Hum Gene Ther. 2012;23(5):533-539. 
15. Calcedo R, Wilson JM. AAV natural infection induces broad cross-neutralizing antibody responses to multiple AAV serotypes in chimpanzees. Hum Gene Ther Clin Dev. 2016;27(2):79-82.

16. Chirmule $\mathrm{N}$, et al. Humoral immunity to adeno-associated virus type 2 vectors following administration to murine and nonhuman primate muscle. $J$ Virol. 2000;74(5):2420-2425.

17. Nathwani AC, et al. Safe and efficient transduction of the liver after peripheral vein infusion of self-complementary AAV vector results in stable therapeutic expression of human FIX in nonhuman primates. Blood. 2007;109(4):1414-1421.

18. Nathwani AC, et al. Adenovirus-associated virus vector-mediated gene transfer in hemophilia B. N Engl J Med. 2011;365(25):2357-2365.

19. Barzel A, et al. Promoterless gene targeting without nucleases ameliorates haemophilia B in mice. Nature. 2015;517(7534):360-364

20. Porro F, et al. Promoterless gene targeting without nucleases rescues lethality of a Crigler-Najjar syndrome mouse model. EMBO Mol Med. 2017;9(10):1346-1355.

21. Carroll D. Genome engineering with targetable nucleases. Annu Rev Biochem. 2014;83:409-439.

22. Rouet P, Smih F, Jasin M. Expression of a site-specific endonuclease stimulates homologous recombination in mammalian cells Proc Natl Acad Sci U S A. 1994;91(13):6064-6068.

23. Xue W, et al. CRISPR-mediated direct mutation of cancer genes in the mouse liver. Nature. 2014;514(7522):380-384.

24. Yin $\mathrm{H}$, et al. Genome editing with Cas9 in adult mice corrects a disease mutation and phenotype. Nat Biotechnol. 2014;32(6):551-553.

25. Yin $\mathrm{H}$, et al. Therapeutic genome editing by combined viral and non-viral delivery of CRISPR system components in vivo. Nat Biotechnol. 2016;34(3):328-333.

26. Yang Y, et al. A dual AAV system enables the Cas9-mediated correction of a metabolic liver disease in newborn mice. Nat Biotechnol. 2016;34(3):334-338.

27. Pankowicz FP, et al. Reprogramming metabolic pathways in vivo with CRISPR/Cas9 genome editing to treat hereditary tyrosinaemia. Nat Commun. 2016;7:12642.

28. Ran FA, et al. In vivo genome editing using Staphylococcus aureus Cas9. Nature. 2015;520(7546):186-191.

29. Bortolussi G, Baj G, Vodret S, Viviani G, Bittolo T, Muro AF. Age-dependent pattern of cerebellar susceptibility to bilirubin neurotoxicity in vivo in mice. Dis Model Mech. 2014;7(9):1057-1068.

30. Bortolussi G, et al. Rescue of bilirubin-induced neonatal lethality in a mouse model of Crigler-Najjar syndrome type I by AAV9-mediated gene transfer. FASEB J. 2012;26(3):1052-1063.

31. Asokan A, Schaffer DV, Samulski RJ. The AAV vector toolkit: poised the clinical crossroads. Mol Ther. 2012;20(4):699-708

32. Porro F, Bockor L, De Caneva A, Bortolussi G, Muro AF. Generation of Ugt1-deficient murine liver cell lines using TALEN technology. PLoS One. 2014;9(8):e104816.

33. Canu G, Minucci A, Zuppi C, Capoluongo E. Gilbert and Crigler Najjar syndromes: an update of the UDP-glucuronosyltransferase 1A1 (UGT1A1) gene mutation database. Blood Cells Mol Dis. 2013;50(4):273-280.

34. Kadakol A, Ghosh SS, Sappal BS, Sharma G, Chowdhury JR, Chowdhury NR. Genetic lesions of bilirubin uridine-diphosphoglucuronate glucuronosyltransferase (UGT1A1) causing Crigler-Najjar and Gilbert syndromes: correlation of genotype to phenotype. Hum Mutat. 2000;16(4):297-306.

35. Caldovic L, Abdikarim I, Narain S, Tuchman M, Morizono H. Genotype-phenotype correlations in ornithine transcarbamylase deficiency: a mutation update. J Genet Genomics. 2015;42(5):181-194.

36. Tuchman M, Jaleel N, Morizono H, Sheehy L, Lynch MG. Mutations and polymorphisms in the human ornithine transcarbamylase gene. Hum Mutat. 2002;19(2):93-107.

37. Sadelain M, Papapetrou EP, Bushman FD. Safe harbours for the integration of new DNA in the human genome. Nat Rev Cancer. 2011;12(1):51-58.

38. Bortolussi G, Muro AF. Advances in understanding disease mechanisms and potential treatments for Crigler-Najjar syndrome. Expert Opin Orphan Drugs. 2018;6(7):425-439.

39. Ronzitti G, et al. A translationally optimized AAV-UGT1A1 vector drives safe and long-lasting correction of Crigler-Najjar syndrome. Mol Ther Methods Clin Dev. 2016;3:16049.

40. Collaud F, et al. Preclinical development of an AAV8-hUGT1A1 vector for the treatment of Crigler-Najjar Syndrome. Mol Ther Methods Clin Dev. 2019;12:157-174.

41. Bočkor L, et al. Repeated AAV-mediated gene transfer by serotype switching enables long-lasting therapeutic levels of hUgtla1 enzyme in a mouse model of Crigler-Najjar Syndrome Type I. Gene Ther. 2017;24(10):649-660

42. Greig JA, Nordin JML, Draper C, Bell P, Wilson JM. AAV8 gene therapy rescues the newborn phenotype of a mouse model of Crigler-Najjar. Hum Gene Ther. 2018;29(7):763-770

43. Sharma R, et al. In vivo genome editing of the albumin locus as a platform for protein replacement therapy. Blood. 2015;126(15):1777-1784.

44. White GC, et al. Definitions in hemophilia. Recommendation of the scientific subcommittee on factor VIII and factor IX of the scientific and standardization committee of the International Society on Thrombosis and Haemostasis. Thromb Haemost 2001;85(3):560.

45. Fox IJ, et al. Treatment of the Crigler-Najjar syndrome type I with hepatocyte transplantation. $N$ Engl J Med. 1998;338(20):1422-1426.

46. Sneitz N, Bakker CT, de Knegt RJ, Halley DJ, Finel M, Bosma PJ. Crigler-Najjar syndrome in The Netherlands: identification of four novel UGT1A1 alleles, genotype-phenotype correlation, and functional analysis of 10 missense mutants. Hum Mutat. 2010;31(1):52-59.

47. Briand P, Francois B, Rabier D, Cathelineau L. Ornithine transcarbamylase deficiencies in human males. Kinetic and immunochemical classification. Biochim Biophys Acta. 1982;704(1):100-106.

48. Ceccaldi R, Rondinelli B, D'Andrea AD. Repair pathway choices and consequences at the double-strand break. Trends Cell Biol. 2016;26(1):52-64

49. Friedland AE, et al. Characterization of Staphylococcus aureus Cas9: a smaller Cas9 for all-in-one adeno-associated virus delivery and paired nickase applications. Genome Biol. 2015;16:257. 
50. Casini A, et al. A highly specific SpCas9 variant is identified by in vivo screening in yeast. Nat Biotechnol. 2018;36(3):265-271.

51. Slaymaker IM, Gao L, Zetsche B, Scott DA, Yan WX, Zhang F. Rationally engineered Cas9 nucleases with improved specificity. Science. 2016;351(6268):84-88.

52. Kleinstiver BP, et al. High-fidelity CRISPR-Cas9 nucleases with no detectable genome-wide off-target effects. Nature. 2016;529(7587):490-495.

53. Kim S, Kim D, Cho SW, Kim J, Kim JS. Highly efficient RNA-guided genome editing in human cells via delivery of purified Cas9 ribonucleoproteins. Genome Res. 2014;24(6):1012-1019.

54. Liang X, et al. Rapid and highly efficient mammalian cell engineering via Cas9 protein transfection. J Biotechnol. 2015;208:44-53.

55. Ramakrishna S, Kwaku Dad AB, Beloor J, Gopalappa R, Lee SK, Kim H. Gene disruption by cell-penetrating peptide-mediated delivery of Cas9 protein and guide RNA. Genome Res. 2014;24(6):1020-1027.

56. Petris G, et al. Hit and go CAS9 delivered through a lentiviral based self-limiting circuit. Nat Commun. 2017;8:15334.

57. Merienne N, et al. The self-inactivating KamiCas9 system for the editing of CNS disease genes. Cell Rep. 2017;20(12):2980-2991.

58. Kosicki M, Tomberg K, Bradley A. Repair of double-strand breaks induced by CRISPR-Cas9 leads to large deletions and complex rearrangements. Nat Biotechnol. 2018;36(8):765-771.

59. Guschin DY, Waite AJ, Katibah GE, Miller JC, Holmes MC, Rebar EJ. A rapid and general assay for monitoring endogenous gene modification. Methods Mol Biol. 2010;649:247-256.

60. Vodret S, et al. Albumin administration prevents neurological damage and death in a mouse model of severe neonatal hyperbilirubinemia. Sci Rep. 2015;5:16203.

61. Bae S, Park J, Kim JS. Cas-OFFinder: a fast and versatile algorithm that searches for potential off-target sites of Cas9 RNA-guided endonucleases. Bioinformatics. 2014;30(10):1473-1475.

62. Li H, Durbin R. Fast and accurate short read alignment with Burrows-Wheeler transform. Bioinformatics. 2009;25(14):1754-1760

63. Li H. A statistical framework for SNP calling, mutation discovery, association mapping and population genetical parameter estimation from sequencing data. Bioinformatics. 2011;27(21):2987-2993.

64. McKenna A, et al. The Genome Analysis Toolkit: a MapReduce framework for analyzing next-generation DNA sequencing data. Genome Res. 2010;20(9):1297-1303. 